\title{
La explotación de moluscos intermareales durante el Holoceno tardío en la localidad de Heshkaia, bahía Moat, sur de Tierra del Fuego
}

\author{
Suray A. Pérez \\ Recibido 11 de julio 2019. Aceptado 25 de noviembre 2019
}

\begin{abstract}
RESUMEN
Se analiza el registro arqueomalacológico de la localidad de Heshkaia, ubicada en la boca oriental del canal Beagle. Mediante estudios de composición taxonómica y biométricos, se evalúa la incidencia de factores antrópicos y ambientales en la configuración de los conjuntos de valvas de tres sitios arqueológicos con fechados del Holoceno tardío. Los resultados indican la recolección en racimos de mejillones (Mytilus edulis) de tamaño subóptimo durante toda la secuencia de ocupación en la localidad. Se infiere que la importancia de los moluscos en la subsistencia humana se definió por su abundancia y accesibilidad, antes que por el aporte alimentario. Asimismo, los cambios temporales en el tamaño de algunos taxones no pueden ser explicados por las fluctuaciones en la temperatura de la superficie del agua de mar y sugieren en cambio un escenario de constante presión antrópica. Esta última limitó el crecimiento máximo de los moluscos más explotados, pero sin comprometer a largo plazo su reproducción local. Los resultados obtenidos aportan nueva información sobre las modalidades de explotación de moluscos y permiten explorar continuidades y diferencias con respecto a los trabajos previos en la costa sur de Tierra del Fuego.
\end{abstract}

Palabras clave: Cazadores-recolectores; Arqueomalacología; Tierra del Fuego; Conchales; Holoceno tardío.

\begin{abstract}
INTERTIDAL MOLLUSC EXPLOITATION DURING THE LATE HOLOCENE AT THE HESHKAIA LOCALITY, BAHÍA MOAT, SOUTHERN TIERRA DEL FUEGO. This paper examines the archaeomalacological record of the Heshkaia locality, situated at the eastern mouth of Beagle Channel. Taxonomic identification and biometric analysis of shells from three Late-Holocene assemblages are performed to assess the incidence of anthropic and natural forces in the observed trends. Results support a shellfish gathering strategy centered on suboptimal blue mussels (Mytilus edulis) stripping along the occupational sequence in the locality. It is inferred that the importance of mollusks in human subsistence derived from their abundance and accessibility, rather than their nutritional contribution. In addition, temporal changes in mean shell size of some species cannot be explained by sea surface temperature variations and instead a constant human harvest pressure scenario is suggested. Although this limited the maximum length mollusks could grow, it did not affect their long-term local reproduction. These results contribute new information on mollusk exploitation patterns and allow examination of continuities and differences with previous studies in the south coast of Tierra del Fuego.
\end{abstract}

Keywords: Hunter-gatherers; Archaeomalacology; Tierra del Fuego; Shell middens; Late-Holocene.

Suray A. Pérez. Laboratorio de Antropología Centro Austral de Investigaciones Científicas. Consejo Nacional de Investigaciones Científicas y Técnicas (CADIC-CONICET). Bernardo Houssay 200 (V94I0CAB), Ushuaia, Tierra del Fuego. E-mail: surayperez@cadic-conicet.gob.ar

Intersecciones en Antropología 21(1), enero-junio: 43-55. 2020. ISSN-e 1850-373X https://doi.org/10.37176/iea.21.1.2020.529

Facultad de Ciencias Sociales - UNICEN - Argentina 


\section{INTRODUCCIÓN}

El conocimiento sobre el uso humano de los recursos malacológicos en la región del canal Beagle durante el Holoceno descansa esencialmente en los estudios efectuados por Orquera (1999) y Orquera y Piana $(2000,2001)$ en su sector central. Allí, los autores identificaron una estrategia de explotación oportunista de estos invertebrados, centrada en mejillones de la especie Mytilus edulis. Debido a su bajo rendimiento calórico, estos han sido interpretados como recursos complementarios en la dieta y como factores reductores de riesgo ante la eventual imposibilidad de acceder a otros recursos de mayor porte, como guanacos y pinnípedos (Orquera, 1999).

Por otro lado, trabajos previos plantean una dinámica de poblaciones humanas que en el pasado integró distintos ambientes de la franja sur de Tierra del Fuego, lo que implicaría diferencias espaciales en el aprovechamiento del entorno costero (Zangrando et al., 2009, 2010). Este escenario conduce a considerar el registro arqueomalacológico desde un enfoque espacial amplio. No obstante, algunos sectores de la costa sur de Tierra del Fuego, y principalmente su porción más oriental, presentan escasa información sobre los restos arqueológicos de moluscos. El estudio inicial del sitio 35 de la localidad de Heshkaia, ubicada en bahía Moat (Figura 1) muestra una continuidad con el canal Beagle central en cuanto a la explotación preferencial de mejillones por los grupos humanos del Holoceno tardío (Zangrando et al., 2017). Los datos del sitio indican un patrón de recolección centrado en mitílidos pequeños (largo promedio 31,7 $\pm 7,1 \mathrm{~mm})$ y de muy bajo rendimiento alimenticio. Esto ha sido hipotéticamente asociado a un proceso local de disminución en la disponibilidad de estos recursos $\mathrm{O}$, alternativamente, a las ligeras fluctuaciones en la temperatura del agua del canal Beagle registradas en los últimos 1000 años (Zangrando et al., 2017) y asociadas a eventos climáticos como la Anomalía
Climática Medieval y la Pequeña Edad de Hielo (Obelic et al., 1998; Gordillo et al., 2015).

En este trabajo se analiza en detalle la secuencia arqueológica de la localidad de Heshkaia y se evalúa la incidencia de factores antrópicos y ambientales en la composición taxonómica y tamaño de los exoesqueletos calcáreos de los conjuntos malacológicos. Asimismo, puesto que las características fisiográficas y ecológicas de dicha localidad contrastan en múltiples aspectos con aquellas de la porción central del canal Beagle, se exploran las similitudes y diferencias en la composición de los conjuntos arqueomalacológicos tardíos de ambos sectores.

\section{ENFOQUE TEÓRICO-METODOLÓGICO}

Las modalidades de explotación humana de moluscos durante el Holoceno tardío son abordadas mediante un análisis que combina la aplicación de modelos de aprovisionamiento óptimo con variables ecológicas y biológicas de estos recursos. En dicho análisis se incorporan datos actuales sobre la malacofauna local y regional publicados en Orquera (1999), Ojeda et al. (2014) y Zangrando et al. (2017).

De acuerdo con los lineamientos del modelo de selección de presas, se plantea que en los sitios de Heshkaia existió una estrategia optimizadora de recolección selectiva de los taxones y ejemplares de moluscos más grandes y de mayor rendimiento energético, conocida como estrategia plucking

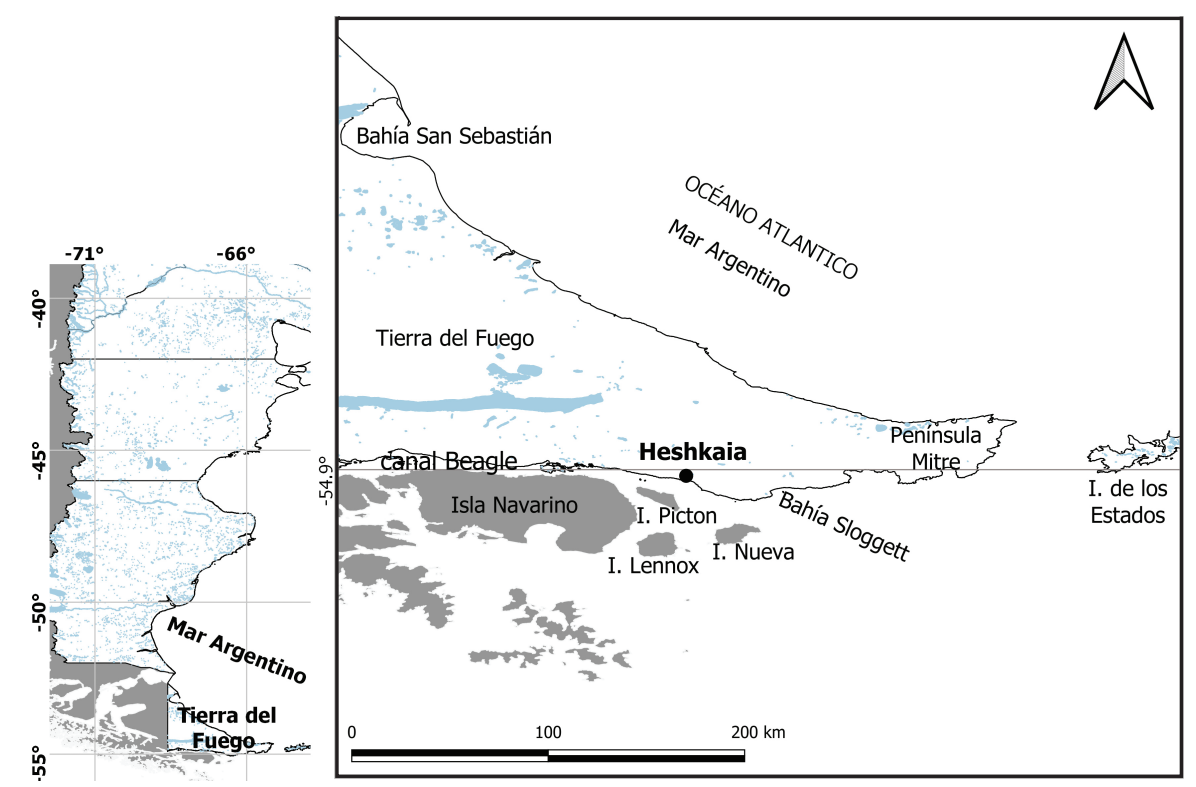

Figura 1. Ubicación geográfica de la localidad arqueológica de Heshkaia. 
(Jones y Richman, 1995). No obstante, se contempla la posibilidad de prácticas de recolección en racimos o del tipo stripping, registradas en algunos contextos etnográficos y arqueológicos (Jones y Richman, 1995; Whitaker, 2008). Estas producen una acumulación de exoesqueletos con amplia variabilidad de tamaños, similar a la de una población natural sin alteración antrópica.

Las predicciones del modelo del lugar central pueden ser evaluadas a partir de la proporción carne-valva de cada taxón y de la distancia a los lugares de consumo (Bettinger y Malhi, 1997; Codding et al., 2014). Dentro de un radio de aprovisionamiento dado, los taxones con una baja proporción carne-valva cuentan con mayor probabilidad de ser procesados en los lugares de recolección. No obstante, estas predicciones no aplican en un contexto de recolección en racimos, en cuyo caso la explotación no estaría condicionada por principios de optimización (Bettinger y Malhi, 1997).

Por otro lado, la distribución heterogénea de los moluscos a lo largo del gradiente vertical y horizontal del intermareal implica diferencias en las tasas de encuentro de los distintos taxones (Ojeda et al., 2014; Zangrando et al., 2017). Asimismo, la historia de vida de cada taxón define en buena medida su resiliencia al impacto antrópico. Generalmente, los moluscos más grandes y relativamente móviles, como algunas especies de gasterópodos, cuentan con tasas de maduración y reproducción más lentas que las especies sésiles, gregarias y de pequeño tamaño, lo cual los hace más vulnerables a la sobreexplotación humana (Stiner et al., 2000; Whitaker y Byrd, 2014). Recolecciones muy intensivas pueden alterar la estructura poblacional y abundancia natural de los moluscos, por lo que se asume que su sobreexplotación involucraría un proceso de disminución diacrónica en el tamaño y abundancia relativa de los taxones más consumidos (Mannino y Thomas, 2002).

El desarrollo de las poblaciones de moluscos también puede verse afectado por procesos ambientales locales y/o regionales asociados a eventos de cambio climático de corta y larga duración. Algunos estudios en ecología han identificado una correlación entre las fluctuaciones térmicas en el agua de mar superficial y los cambios en las tasas de crecimiento de los moluscos (Menge et al., 2008). Las oscilaciones registradas en el tamaño de bivalvos del género Mytilus durante las fases climáticas del ENOS sugieren que incluso las especies euritópicas pueden manifestar cambios en sus tasas de crecimiento ante fluctuaciones ambientales. Tales cambios constituirían una respuesta integrada al efecto directo de la temperatura y al efecto indirecto de la disponibilidad de alimento (Menge et al., 2008). Esta información plantea entonces la necesidad de considerar la influencia de dichos factores en la variación de los conjuntos arqueomalacológicos de la región del canal Beagle. Allí, Obelic et al. (1998) y Gordillo et al. (2015) han registrado entre los 1000-500 años AP un período de temperaturas más elevadas en las aguas superficiales del canal, que coincide con momentos de la Anomalía Climática Medieval. El aumento en la temperatura $-y$ posiblemente en la productividad primaria de dichas aguas (Gordillo et al., 2015)- habría favorecido un incremento en las tasas de crecimiento de los moluscos (Pérez, 2018). En contraste, entre los 500100 años $\mathrm{AP}$, los autores detectan condiciones de temperatura más frías, que se correlacionan con el evento climático de la Pequeña Edad de Hielo. Bajo dichas condiciones, es posible que los moluscos desarrollaran tamaños más pequeños con respecto a los momentos previos debido al menor aporte de agua dulce y de materia orgánica terrestre (Gordillo et al., 2015; Pérez, 2018).

\section{MARCO AMBIENTAL}

La localidad Heshkaia se ubica en bahía Moat, sobre la costa norte del canal Beagle (Zangrando et al., 2010). En este espacio, la cordillera fueguina presenta menor altitud con respecto al sector más occidental de dicha región (Alunni y Zangrando, 2012) y la franja costera se encuentra más expuesta a aguas abiertas y a los vientos del océano Atlántico Sur.

El ambiente costero de la localidad presenta playas extensas de guijarros o arena y pendiente suave, alternadas con abruptos acantilados rocosos. En las aguas someras de estos últimos se desarrollan densos bosques de macroalgas bentónicas Macrocystis pyrifera, que sustentan una gran diversidad de organismos intermareales, entre ellos, moluscos de las clases Bivalvia, Gastropoda y Poliplacophora.

Los distintos taxones de la malacofauna local presentan una distribución vertical y horizontal diferente, asociada a los ciclos de marea, a las características del sustrato, a la penetración de la luz y a la exposición al oleaje (Ojeda et al., 2014; Zangrando et al., 2017). Predominan los filtradores 
sésiles como Mytilus edulis y Brachidontes sp., que suelen establecerse en forma intercalada en plataformas rocosas de abrasión formando densos bancos de mitílidos (Zangrando et al., 2017). Estos moluscos abarcan un rango espacial amplio, que incluye la franja intermareal superior, media e inferior. Los bivalvos de la especie Aulacomya atra aparecen, en cambio, en bajas frecuencias y con tamaños más pequeños que los que suelen alcanzar a mayor profundidad (franja submareal). A su turno, moluscos gasterópodos como las lapas Nacella sp. y Fissurella sp., y los caracoles de mar Acanthina monodon y Pareuthria fuscata, se distribuyen en pequeños grupos sobre bloques de roca semiverticales, junto con algunos ejemplares aislados de quitones (clase Poliplacophora) (Zangrando et al., 2017).

Puesto que en la actualidad los moluscos de la localidad Heshkaia no son explotados antrópicamente, los promedios de longitud documentados por Zangrando et al. (2017) y Pérez (2018) para ejemplares modernos de Mytilus edulis (41,5 \pm 9,9 $\mathrm{mm})$ y Nacella sp. $(39,8 \pm 6,3 \mathrm{~mm})$ pueden considerarse representativos del tamaño corporal óptimo alcanzable por estos taxones.

\section{CASOS DE ESTUDIO}

Los depósitos de concheros excavados hasta el momento en la localidad-denominados Heshkaia $28,30,34$ y $35-$ cuentan con cronologías del Holoceno tardío. Su composición arqueofaunística se caracteriza por una notable representación de guanacos y de aves y peces costeros, y una mínima presencia de recursos pelágicos (Zangrando et al., 2010). A continuación, se presenta brevemente la información estratigráfica y cronológica de los sitios Heshkaia 28, 30 y 34, cuyos conjuntos arqueomalacológicos se analizan en este trabajo.

\section{Heshkaia $28\left(54^{\circ} 56^{\prime} 50,5^{\prime \prime} \mathrm{S}-66^{\circ} 50^{\prime} 34,2^{\prime \prime} \mathrm{O}\right)$}

Se ubica en un ambiente boscoso sobre el límite occidental de la localidad. El sitio consiste en un domo aislado de $20 \mathrm{~m}^{2}$ de escasa potencia, que se sitúa por encima de un paleoacantilado y a $22 \mathrm{~m}$ de la línea de la costa actual. Presenta un conchero de $17 \mathrm{~cm}$ de potencia máxima, dividido en dos unidades estratigráficas: capas C y F. Su cronología es de $678 \pm 38$ años AP, obtenidos en la capa basal del conchero (capa F).

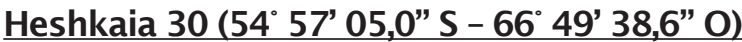

Se emplaza por debajo de un barranco de escasa altura y sobre una playa activa. El sitio consiste en tres montículos alineados que originalmente cubrían una superficie de unos $80 \mathrm{~m}^{2}$ y en cuya base se ha obtenido un fechado de $263 \pm 35$ años AP. Presenta dos unidades de conchales, denominadas $\mathrm{C}$ y $\mathrm{F}$, separadas por una fina capa edafizada.

\section{Heshkaia 34 (54 $57^{\prime} 13,9^{\prime \prime}$ S - 66 $\left.46^{\circ} 02,1^{\prime \prime} 0\right)$}

Se ubica al pie de un barranco, sobre una paleoplaya, a $40 \mathrm{~m}$ de la línea de costa actual, y ha sido fechado en la parte intermedia y más potente del conchero en unos $981 \pm 36$ años AP. Está compuesto por cinco montículos y dos estructuras anulares que ocupan una superficie total de aproximadamente unos $1500 \mathrm{~m}^{2}$. El sitio contiene el depósito de conchal más extenso de la localidad, compuesto por dos unidades estratigráficas, denominadas capa C y capa F.

\section{MATERIALES Y MÉTODOS}

De los depósitos de concheros Heshkaia 28, 30 y 34 , se analiza la composición malacológica de las unidades estratigráficas indicadas en la Tabla 1. La separación entre las capas de cada sitio se asocia exclusivamente a criterios metodológicos de la excavación. Los remontajes de restos faunísticos entre diferentes niveles estratigráficos (Martinoli, 2018) y la ausencia de suelos $u$ otros indicadores de estabilidad descartan la necesidad de diferenciar cronológicamente los conjuntos de moluscos en niveles intrasitio. Los restos malacológicos provienen de muestras sedimentarias de $4 \mathrm{dm}^{3}$ (4 litros) procesadas en el laboratorio según los criterios explicitados por Orquera y Piana $(2000,2001)$. En los sitios Heshkaia 28 y 34, dichas muestras proceden de las subunidades estratigráficas de excavación, mientras que en Heshkaia 30, donde solo se ha

\begin{tabular}{|c|c|c|c|c|}
\hline Sitio & $\begin{array}{c}\text { Clase } \\
\text { de muestra }\end{array}$ & $\begin{array}{c}\text { Referencia } \\
\text { estratigráfica } \\
\text { (capa) }\end{array}$ & $\begin{array}{c}\text { Volumen } \\
\text { de muestra } \\
\left(\mathrm{dm}^{3}\right)\end{array}$ & $\begin{array}{c}\text { Peso } \\
\text { de muestra } \\
(\mathrm{g})\end{array}$ \\
\hline \multirow{3}{*}{ Heshkaia 28} & \multirow{3}{*}{$\begin{array}{c}\text { Muestras } \\
\text { de subunidad }\end{array}$} & $\mathrm{C}$ & 7,6 & 5066,2 \\
\hline & & $\mathrm{F}$ & 9,8 & 6952,4 \\
\hline & & Total & 17,4 & $12.018,6$ \\
\hline Heshkaia 30 & $\begin{array}{c}\text { Muestras } \\
\text { de columna }\end{array}$ & $\mathrm{F}$ & 16,4 & $10.019,2$ \\
\hline \multirow{3}{*}{ Heshkaia 34} & \multirow{3}{*}{$\begin{array}{c}\text { Muestras } \\
\text { de subunidad }\end{array}$} & $\mathrm{C}$ & 11,5 & 9684,8 \\
\hline & & $\mathrm{F}$ & 30 & $24.781,2$ \\
\hline & & Total & 41,5 & $34.466,0$ \\
\hline
\end{tabular}

Tabla 1. Procedencia de las muestras de moluscos analizadas. 
efectuado un sondeo de $1 \times 1 \mathrm{~m}$, estas se obtuvieron de una columna de muestreo excavada en uno de sus perfiles.

Para evaluar el estado de preservación de las muestras arqueomalacológicas, se calculó por sitio un índice de fragmentación que expresa la relación entre el peso de los fragmentos no identificados y el peso de la totalidad de restos de moluscos (identificados y sin identificar) (Zangrando et al., 2017). El índice se evalúa en una escala de 0 a 1 : valores cercanos a 0 indican conjuntos con poca fragmentación; y valores cercanos a 1, conjuntos muy fragmentados.

Los restos de moluscos fueron identificados en la menor categoría taxonómica posible mediante la observación de atributos diagnósticos en las valvas (Gordillo, 1995; Forcelli, 2000).

El número de restos (NR) y el número mínimo de individuos (NMI) fueron estimados a partir de especímenes enteros y fragmentados. Para la determinación de este último se identificaron los elementos no repetitivos (Mason et al., 1998; Verdún Castelló, 2014). El NMI de los moluscos bivalvos se calculó sobre la base del número más alto de charnelas por lateralidad. En gasterópodos, se contabilizaron los ápices y anillos exteriores enteros de las lapas, y los ápices y columelas de los caracoles marinos. Para los poliplacóforos se computaron las placas cefálicas y las caudales. De no haberse hallado estas últimas, se dividió el número de placas intermedias enteras de cada muestra por la cantidad total de placas intermedias que estos moluscos poseen naturalmente (seis).

Por otra parte, se realizó un análisis biométrico en moluscos Mytilus edulis y Nacella sp. Los primeros alcanzan la madurez sexual al año de edad (Lasta et al., 1998; Oehrens Kissner y Kroeck, 2005), mientras que para gasterópodos de gran tamaño como las lapas se estima que aquella ocurriría entre los 2 y 5 años de edad (Stiner et al., 2000). El análisis incluyó ejemplares arqueológicos y modernos, estos últimos, recolectados en un mismo sector del intermareal de la localidad. En individuos completos de Nacella sp., se midió la altura (Al), el ancho máximo (An) y el largo total (Lt)
(Verdún Castelló, 2014; Hammond, 2015), aunque solo se consideró a esta última como parámetro central del tamaño (Figura 2).

Debido a las altas tasas de fragmentación que normalmente presentan los especímenes del género Mytilus (Orquera y Piana, 2001; Campbell, 2012), en este estudio se aplicaron fórmulas alométricas de regresión. Estas permiten estimar la longitud máxima de un individuo a partir de diferentes medidas tomadas en torno al umbo de un espécimen fragmentado. En particular, se utilizó una de las ecuaciones propuestas por Campbell (2012) para la especie Mytilus edulis, recientemente aplicada por Zangrando et al. (2017) en ejemplares arqueológicos de mejillones del canal Beagle:

$$
[\mathrm{L}=2,76471+5,97086 * 1 / 2 \mathrm{Wam}]
$$

donde $L$ representa la longitud máxima de la valva y $1 / 2$ Wam corresponde al ancho del umbo (Figura 2).

El manejo estadístico de los resultados biométricos se efectuó mediante el programa informático PAST 3.23. La normalidad de la distribución de longitudes de mejillones y lapas de cada sitio fue evaluada mediante el Test Shapiro-Wilk. Para la comparación intersitio de los datos se contemplaron

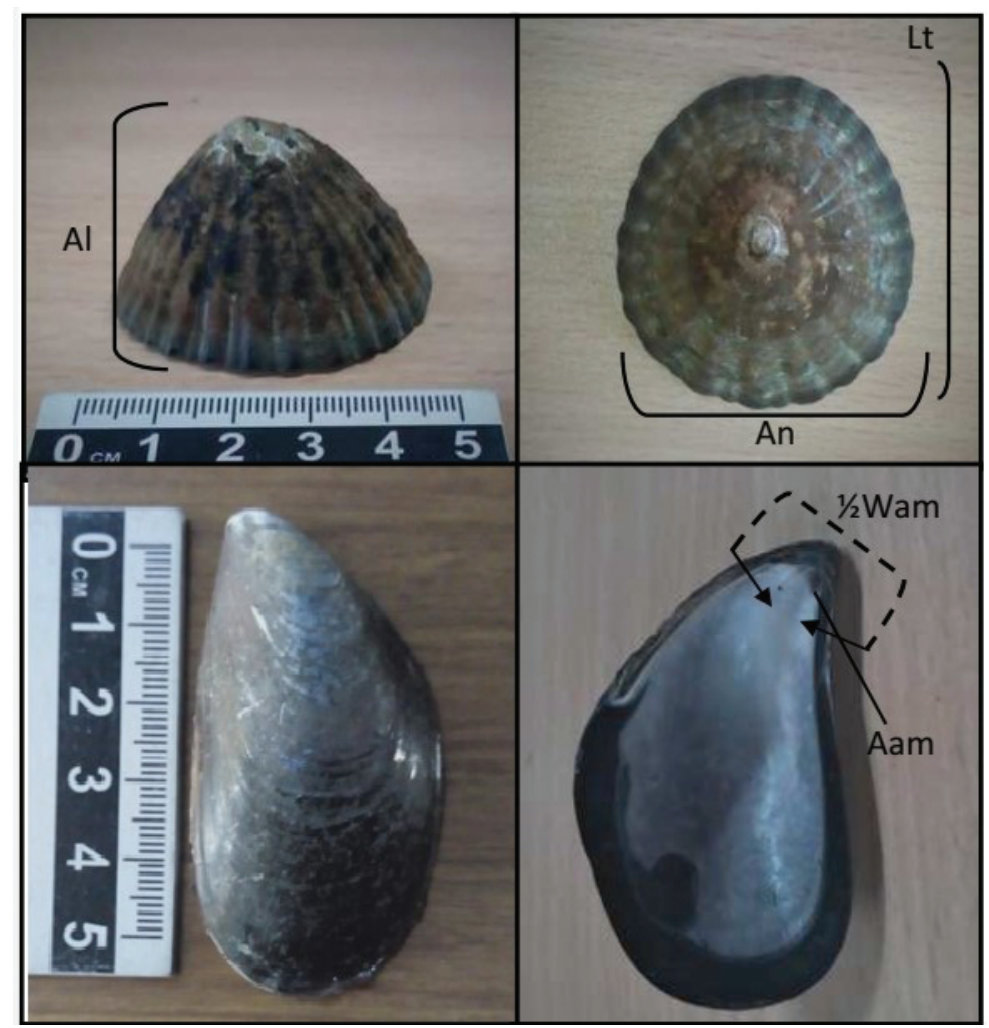

Figura 2. Dimensiones medidas en ejemplares de Nacella sp. (arriba) y Mytilus edulis (abajo). Referencias: alto (Al), ancho (An) y largo (Lt) de especímenes completos de Nacella sp.; impresión del músculo aductor anterior (Aam) y ancho del umbo (1/2 Wam). 
el análisis de varianza (ANOVA), cuando todas las distribuciones fuesen normales, y el método no paramétrico Kruskal-Wallis para el caso contrario, y se utilizó un nivel de significación $p<, 05$. Debido a la recurrente fragmentación de los exoesqueletos calcáreos, se estableció un mínimo de 25 observaciones por muestra de sitio como criterio válido para su inclusión en el análisis comparativo.

Para evaluar la incidencia de factores ambientales, los datos biométricos de los sitios fueron confrontados con las condiciones paleoambientales del canal Beagle descriptas por Obelic et al. (1998) y Gordillo et al. (2015), y correlacionadas con la Anomalía Climática Medieval y la Pequeña Edad de Hielo.

Asimismo, como un intento de profundizar el estudio sobre la influencia de factores antrópicos en la composición malacológica de los conchales de Heshkaia, se efectuaron muestreos y análisis de las acumulaciones naturales de restos de moluscos depositados en las playas de dicha localidad. Los conjuntos de valvas, placas y conchillas vacías se recolectaron a partir de cuatro cuadrantes de $1 \times 1$ $\mathrm{m}$ tomados al azar en la zona supralitoral e intermareal superior de tres sectores costeros próximos a los sitios arqueológicos. En general, los ensambles naturales fueron encontrados en las bermas de tormenta de playas compuestas por grava y por guijarros y arena. Su caracterización se efectuó a partir de los mismos parámetros analizados en los depósitos arqueológicos a fines de facilitar la comparación: índice de fragmentación, diversidad y abundancia taxonómica. emplaza implicarían condiciones más ácidas y desfavorables para la preservación de la estructura calcárea de los moluscos. Más allá de estas diferencias, los tres depósitos arqueológicos presentan índices de fragmentación superiores a aquellos de los ensambles naturales de restos malacológicos, los cuales registran valores mínimos que fluctúan entre 0,05 y 0,11 .

Aunque la cantidad total de restos identificados varía por sitio según las condiciones de preservación, en la Tabla 2 se observa que moluscos de valvas frágiles como los Mytilus edulis mantienen en todos los casos el mismo orden de importancia. Asimismo, la densidad de restos de taxones con valvas más robustas (i.e., Brachidontes sp. y Acanthina monodon) difiere poco entre los conjuntos. Esto indica que la fragmentación no alteró sustancialmente la relación entre taxones dentro de los conjuntos de moluscos, lo que posibilita la comparación intersitio de la composición malacológica.

En la Tabla 3 se presentan las frecuencias de NMI de los taxones registrados en cada conjunto arqueomalacológico. La diversidad taxonómica es bastante similar entre las muestras de cada sitio. En todos los casos, los bivalvos constituyen la clase de moluscos más abundante; entre ellos, Mytilus edulis constituye la especie dominante, con frecuencias de $\mathrm{NMI}$ muy superiores a las del resto de los taxones, especialmente en Heshkaia 30. Siguen en orden de importancia los mitílidos Brachidontes sp. y las lapas del género Nacella. Los restantes taxones de la clase Gastropoda (Fissurella sp., Siphonaria sp. y Pareuthria fuscata) y Poliplacophora aparecen siempre en muy bajas proporciones.

\section{RESULTADOS}

De los conjuntos de moluscos analizados, el sitio más reciente, Heshkaia 30, registra las mejores condiciones de preservación, con un índice de fragmentación de 0,44. Los sitios 28 y 34 presentan valores más elevados de 0,74 y 0,81 , respectivamente. Posiblemente, la menor preservación de los restos malacológicos de Heshkaia 34 responda a un factor cronológico, dado que constituye el conjunto arqueológico más temprano de la localidad. En cuanto a Heshkaia 28, la escasa potencia del conchero y el ambiente boscoso en el que se

\begin{tabular}{|c|c|c|c|c|c|c|c|}
\hline & & \multicolumn{2}{|c|}{ Heshkaia 30} & \multicolumn{2}{|c|}{ Heshkaia 28} & \multicolumn{2}{|c|}{ Heshkaia 34} \\
\hline & & NR & $\mathrm{NR} / \mathrm{dm}^{3}$ & NR & $\mathrm{NR} / \mathrm{dm}^{3}$ & NR & $\mathrm{NR} / \mathrm{dm}^{3}$ \\
\hline \multirow{3}{*}{$\frac{\frac{\pi}{2}}{\frac{\pi}{2}}$} & Mytilus edulis & 4027 & 245,5 & 1187 & 67,8 & 3458 & 83,3 \\
\hline & Brachidontes sp. & 213 & 13,0 & 212 & 12,1 & 421 & 10,1 \\
\hline & Aulacomya atra & 34 & 2,1 & 0 & 0 & 0 & 0 \\
\hline \multirow{5}{*}{ 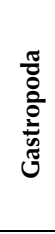 } & Nacella sp. & 198 & 12,1 & 45 & 2,6 & 124 & 3,0 \\
\hline & Siphonaria sp. & 22 & 1,3 & 22 & 1,3 & 103 & 2,5 \\
\hline & Fissurella sp. & 5 & 0,3 & 7 & 0,4 & 93 & 2,2 \\
\hline & Acanthina monodon & 230 & 14,0 & 191 & 10,9 & 166 & 4,0 \\
\hline & Pareuthria fuscata & 0 & 0 & 0 & 0 & 5 & 0,1 \\
\hline & Poliplacophora & 47 & 2,9 & 49 & 2,8 & 268 & 6,5 \\
\hline \multicolumn{2}{|c|}{ Total } & 4776 & 291,2 & 1713 & 97,9 & 4638 & 111,8 \\
\hline
\end{tabular}

Tabla 2. Número (NR) y densidad $\left(\mathrm{NR} / \mathrm{dm}^{3}\right)$ de restos de los taxones identificados por sitio. Los valores de densidad fueron calculados a partir del volumen global de la muestra de cada sitio especificado en la Tabla 1. 


\begin{tabular}{|c|c|c|c|c|c|c|c|}
\hline & & \multicolumn{2}{|c|}{ Heshkaia 30} & \multicolumn{2}{|c|}{ Heshkaia 28} & \multicolumn{2}{|c|}{ Heshkaia 34} \\
\hline & & NMI & $\%$ & NMI & $\%$ & NMI & $\%$ \\
\hline \multirow{3}{*}{ 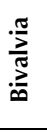 } & Mytilus edulis & 2024 & 83,9 & 581 & 70,9 & 1434 & 72,7 \\
\hline & Brachidontes sp. & 110 & 4,6 & 108 & 13,2 & 203 & 10,3 \\
\hline & Aulacomya atra & 22 & 0,9 & 0 & 0 & 0 & 0 \\
\hline \multirow{5}{*}{ 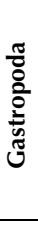 } & Nacella sp. & 198 & 8,2 & 45 & 5,5 & 124 & 6,3 \\
\hline & Siphonaria sp. & 22 & 0,9 & 22 & 2,7 & 103 & 5,2 \\
\hline & Fissurella sp. & 2 & 0,1 & 4 & 0,5 & 41 & 2,1 \\
\hline & Acanthina monodon & 28 & 1,2 & 33 & 4,0 & 28 & 1,4 \\
\hline & Pareuthria fuscata & 0 & 0 & 0 & 0 & 5 & 0,3 \\
\hline & Poliplacophora & 6 & 0,2 & 26 & 3,1 & 35 & 1,8 \\
\hline \multicolumn{2}{|c|}{ Total } & 2412 & 100 & 819 & 100 & 1973 & 100 \\
\hline \multicolumn{2}{|c|}{$\mathrm{N}$ - taxones } & 9 & - & 8 & - & 9 & - \\
\hline
\end{tabular}

Tabla 3. Composición taxonómica en los sitios arqueológicos Heshkaia 28,30 y 34 .

La composición de las acumulaciones actuales de restos de moluscos difiere notablemente entre los tres sectores analizados (Tabla 4). Aunque el número de taxones en cada ensamble es inferior al que se registra en los depósitos arqueológicos, el total de géneros y especies identificados a partir de las tres acumulaciones actuales $(n=12)$ es superior y sugiere la existencia de una malacofauna local mucho más diversa.

Respecto del análisis biométrico (Tabla 5), los datos de Heshkaia 28 fueron omitidos en la discusión debido a que la cantidad de mediciones efectuadas en umbos de Mytilus edulis $(n=28)$ y en especímenes completos de Nacella sp. ( $\mathrm{n}=18)$ fue inferior al mínimo establecido para el análisis comparativo.

Todas las muestras de Mytilus edulis -modernas y arqueológicas- registran diferencias estadísticas significativas entre sí (Kruskal-Wallis $p<, 05)$. Los mejillones actuales presentan el tamaño medio más elevado y la mayor variabilidad de medidas de longitud, con ejemplares que miden hasta unos $16 \mathrm{~mm}$ más que los arqueológicos. Aun así, las muestras de individuos de Heshkaia 30 y 34 cuentan con un amplio rango de tamaños, y sus valores centrales se aproximan a los de la muestra actual. Asimismo, se detectan diferencias significativas entre los tamaños de todas las muestras de Nacella sp. $(p<, 05)$, y los ejemplares actuales presentan mayor variabilidad de medidas de longitud y un tamaño promedio superior al de los arqueológicos. actuales.

\section{DISCUSIÓN}

Los depósitos arqueológicos de Heshkaia registran la presencia de taxones característicos de las zonas intermareales de la localidad, cuyos restos se encuentran representados en los ensambles naturales de las playas circundantes. A diferencia de estos últimos, las muestras arqueomalacológicas evidencian niveles de fragmentación notablemente más altos $y$, en conjunto, una menor diversidad de taxones, lo cual es coherente con lo esperado para concentraciones de moluscos que han estado sujetas a procesos de selección y acumulación antrópica. Asimismo, la semejanza general que se detecta en la composición malacológica de Heshkaia 28, 30 y 34 indica que los cazadores-recolectores locales mantuvieron un mismo criterio de selección de taxones a lo largo del tiempo y de forma independiente con respecto al lugar de emplazamiento de los sitios.

\begin{tabular}{|c|c|c|c|c|c|c|c|}
\hline & \multicolumn{2}{|c|}{ Sector 1} & \multicolumn{2}{|c|}{ Sector 2} & \multicolumn{2}{|c|}{ Sector 3} \\
\hline & & NMI & $\%$ & NMI & $\%$ & NMI & $\%$ \\
\hline \multirow{5}{*}{$\frac{\frac{\pi}{2}}{\frac{\pi}{\pi}}$} & Mytilus edulis & 4 & 10,5 & 88 & 61,1 & 5 & 7,0 \\
\hline & Brachidontes sp. & 0 & 0 & 8 & 5,6 & 0 & 0 \\
\hline & Aulacomya ater & 5 & 13,2 & 12 & 8,3 & 5 & 7,0 \\
\hline & Retrotapes exalbidus & 0 & 0 & 0 & 0 & 1 & 1,4 \\
\hline & Tawera gayi & 0 & 0 & 0 & 0 & 1 & 1,4 \\
\hline \multirow{6}{*}{$\begin{array}{l}\frac{\pi}{\circ} \\
\vdots \\
\frac{\vdots}{0} \\
\text { 心 }\end{array}$} & Nacella sp. & 26 & 68,4 & 30 & 20,8 & 58 & 81,7 \\
\hline & Nacella mytilina & 2 & 5,3 & 0 & 0 & 0 & 0 \\
\hline & Fissurella sp. & 0 & 0 & 2 & 1,4 & 0 & 0 \\
\hline & Muricidae sp. & 1 & 2,6 & 0 & 0 & 0 & 0 \\
\hline & Acanthina monodon & 0 & 0 & 3 & 2,1 & 0 & 0 \\
\hline & Crepipatella dilatata & 0 & 0 & 1 & 0,7 & 0 & 0 \\
\hline 总 & Poliplacophora & 0 & 0 & 0 & 0 & 1 & 1,4 \\
\hline \multicolumn{2}{|c|}{ Total } & 38 & - & 144 & - & 71 & - \\
\hline \multicolumn{2}{|c|}{$N^{o}$ taxones } & 5 & - & 7 & - & 6 & - \\
\hline
\end{tabular}

Tabla 4. Composición malacológica en los sitios de muestreo

\begin{tabular}{|c|c|c|c|c|}
\hline \multirow{7}{*}{ 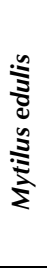 } & & Actuales & Heshkaia 30 & Heshkaia 34 \\
\hline & $\mathrm{N}$ & 453 & 259 & 83 \\
\hline & Mediana $(\mathrm{mm})$ & 39,8 & 36,7 & 38,1 \\
\hline & Rango intercuartil (mm) & $34,3-46,5$ & $32,7-41,0$ & $34,3-41,7$ \\
\hline & Media $(\mathrm{mm})$ & 41,5 & 36,7 & 38,6 \\
\hline & DS \pm & 9,9 & 6,1 & 5,3 \\
\hline & Rango total (mm) & $17,9-70,6$ & $23,0-54,2$ & $28,6-53,9$ \\
\hline \multirow{6}{*}{ 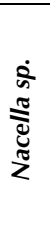 } & $N$ & 122 & 141 & 66 \\
\hline & Mediana $(\mathrm{mm})$ & 40,4 & 37,2 & 34,7 \\
\hline & Rango intercuartil (mm) & $35,8-43,9$ & $33,3-40,4$ & $32,2-37,2$ \\
\hline & Media (mm) & 39,8 & 37 & 34,9 \\
\hline & DS \pm & 6,3 & 5,7 & 5 \\
\hline & Rango total (mm) & $24,9-53,0$ & $13,5-52,3$ & $22,3-49,7$ \\
\hline
\end{tabular}

Tabla 5. Estadística descriptiva de las longitudes totales de ejemplares arqueológicos y actuales de Mytilus edulis y Nacella sp. 
Si se consideran las tasas de rendimiento de la malacofauna local y regional reportadas por Orquera (1999) y Zangrando et al. (2017), se observa que los patrones de representación taxonómica en los depósitos arqueológicos no se ajustan a las expectativas del modelo de amplitud de dieta. Los taxones con mayores tasas de retorno posencuentro -como las lapas Fissurella sp. (7769 kcal/h) y Nacella sp. (2933 kcal/h), o los caracoles Acanthina sp. (2197 $\mathrm{kcal} / \mathrm{h}$ )- se registran en proporciones sensiblemente más bajas que las de los mejillones Mytilus edulis (631 kcal/h). Aunque la proporción carne-valva de estos gasterópodos es superior a la de los mejillones y no justificaría su procesamiento en el locus de recolección (Zangrando et al., 2017), difícilmente pueda defenderse la idea de un transporte diferencial de las partes anatómicas debido a la escasa distancia de los sitios respecto de la línea de costa.

No obstante, en las costas del canal Beagle, los mejillones presentan una abundancia anual y estacional sensiblemente superior a la de otros taxones de moluscos intermareales (Ojeda et al., 2014). También en otras porciones del sistema de canales y fiordos subantárticos se ha registrado su mayor abundancia y plasticidad ecológica (Langley et al., 1980; Rosenfeld et al., 2013). En particular, en los sectores costeros próximos a Heshkaia, esta especie cuenta con las frecuencias de aparición más elevadas (75, 70\%) (Ojeda et al., 2014). En contraste, los moluscos Fissurella sp., Nacella sp., Acanthina monodon y Poliplacophora poseen frecuencias respectivas de 0,69; 14,50; 2,10; y 1,70\% (Ojeda et al., 2014; Zangrando et al., 2017). Si bien no hay información precisa sobre la abundancia local de Aulacomya atra, estos mitílidos habitan preferentemente el ambiente submareal y son menos accesibles durante las mareas normales (Gordillo, 1995). De este modo, la composición malacológica de Heshkaia 28, 30 y 34 coincide con los patrones observados en Heshkaia 35 (Zangrando et al., 2017) y sugiere que las tasas de retorno tuvieron una menor incidencia en los criterios de selección de taxones.

En contraste, las concentraciones naturales de restos malacológicos muestran poca diferencia entre los porcentajes globales de Mytilus edulis $(49,3 \%)$ y los de Nacella sp. (33\%), y se alejan de los patrones de abundancia taxonómica de las comunidades vivientes de moluscos en la región. $\mathrm{Si}$ bien se ha planteado que las asociaciones de valvas vacías mantienen fidelidad ecológica respecto de las comunidades actuales (Kidwell, 2002, 2013), la falta de datos precisos sobre la riqueza y abundancia taxonómica de las poblaciones naturales de Heshkaia impide evaluar su grado de correlación con dichas asociaciones. Esto permitiría detectar y/o descartar posibles sesgos tafonómicos en los resultados del muestreo actualístico (Sandra Gordillo, comunicación personal, 2019).

Las pautas de recolección observadas en la localidad de Heshkaia muestran una línea de continuidad con las del canal Beagle central, para el cual los antecedentes señalan que durante el Holoceno medio y tardío los moluscos fueron recolectados en proporciones similares a su oferta natural (Orquera y Piana, 2001). En dicho sector, sin embargo, los conjuntos más tardíos exhiben una abundancia de mejillones mucho más abrumadora que en Heshkaia, con porcentajes superiores al $98 \%$ en localidades como Túnel, Shamakush y Lancha Packewaia (Figura 3). Esto sugiere la posibilidad de que, junto con una estrategia general de recolección de mitílidos, existiesen algunas variantes espaciales en la importancia del consumo de taxones menos abundantes o rentables. En particular, los Brachidontes sp. se detectan en mínimas frecuencias en los sitios del canal Beagle central y han sido considerados como fauna acompañante y económicamente marginal debido a su pequeña talla, igual o inferior a los $30 \mathrm{~mm}$ (Orquera y Piana, 2001). Ejemplares con longitudes similares se registran en la columna de muestreo del sitio Heshkaia 35 (Zangrando et al., 2013). Sin embargo, en los conjuntos arqueológicos de Heshkaia, estos mitílidos aparecen en proporciones más notables que en aquellos del sector central del canal, lo cual plantea su posible aprovechamiento local.

Por otra parte, el análisis biométrico permitió constatar variaciones en el tamaño promedio de Mytilus edulis y Nacella sp. a lo largo de la secuencia arqueológica local. Sin embargo, dichas variaciones no se correlacionan con las fluctuaciones en la temperatura del agua superficial del canal Beagle detectadas por Obelic et al. (1998) y Gordillo et al. (2015) (Figura 4). Entre los 1000 y los 500 años $\mathrm{AP}$, bajo condiciones favorables para el crecimiento de los moluscos, la especie Mytilus edulis registra en Heshkaia 34 el promedio de tamaño más alto de la secuencia. Al mismo tiempo, en el sitio 35 aparece el tamaño medio más pequeño de toda la secuencia. Posteriormente, entre los 500 y los 100 años $\mathrm{AP}$, y en un supuesto contexto de menor productividad primaria marina, los mejillones del sitio Heshkaia 30 muestran, sin embargo, un incremento en su tamaño. Asimismo, los datos correspondientes 


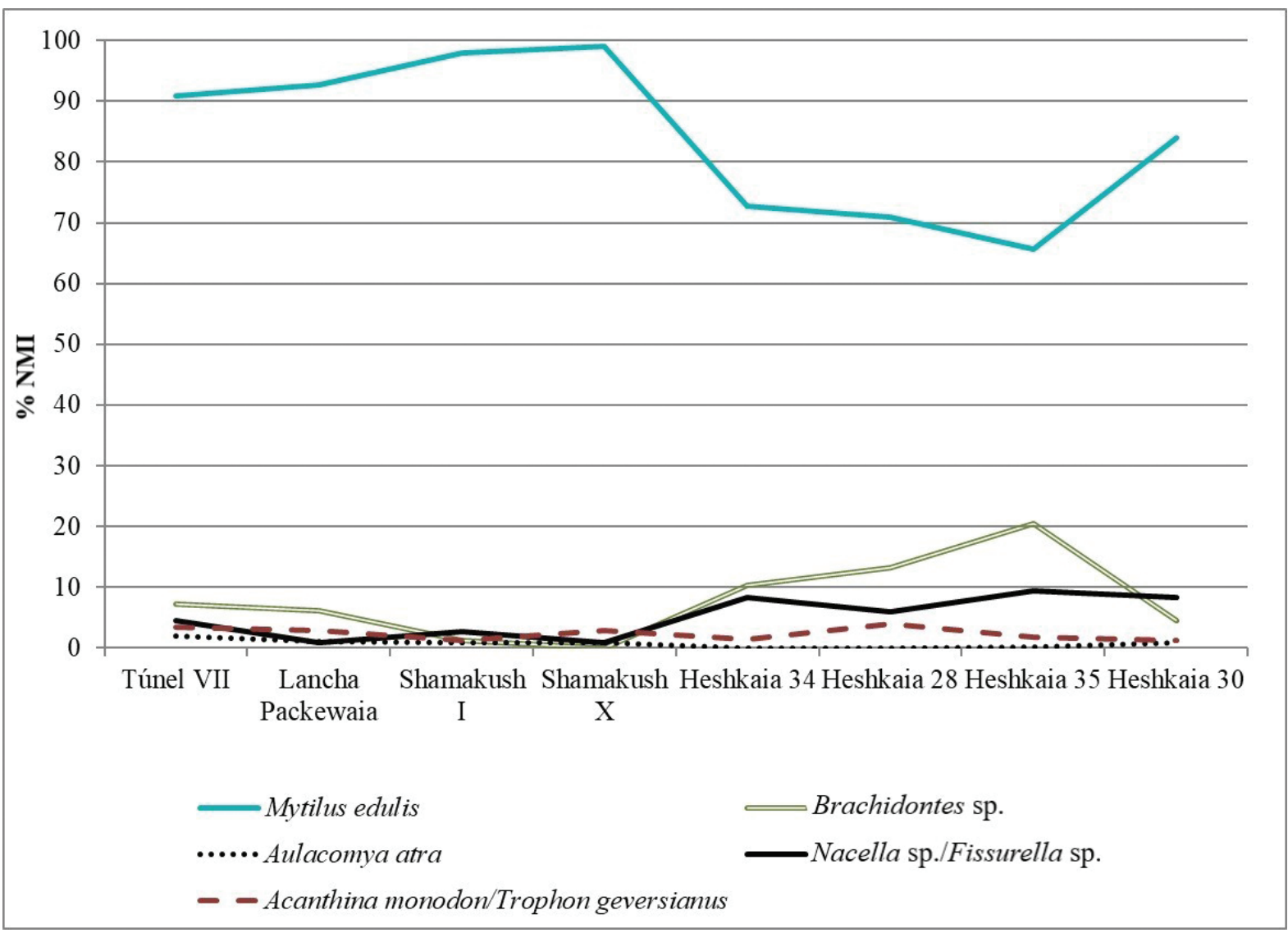

Figura 3. Composición malacológica (\% NMI) en contextos tardíos de diferentes localidades arqueológicas de la costa norte del canal Beagle.

a Nacella sp. sugieren un aumento en el promedio de longitud a lo largo del tiempo, aunque estos no pueden considerarse definitivos dada la falta de información biométrica para la parte media de la secuencia de ocupación (en sitios Heshkaia 28 y 35). Aun así, la totalidad de los resultados obtenidos indica que los cambios en el tamaño de los ejemplares arqueológicos de moluscos no pueden ser explicados en principio por las variaciones térmicas en el agua superficial del canal Beagle registradas en los últimos ca. 1000 años AP.

Las variaciones temporales en la longitud de los exoesqueletos sugieren en cambio un vínculo más directo con las prácticas de recolección de los cazadores-recolectores tardíos de Heshkaia. A lo largo de la secuencia arqueológica local, la presencia continua de moluscos con tamaños inferiores a los desarrollados en el ambiente intermareal actual señala un fenómeno de constante explotación humana que limitaría el crecimiento máximo alcanzable por estos invertebrados. Esto ha sido observado previamente en los contextos tardíos del sector central del canal Beagle, aunque en estos últimos la amplitud de medidas de longitud documentada fue mayor, y en algunos depósitos se detectaron promedios superiores a los obtenidos en este estudio (Orquera y Piana, 2001).

Pese a su mayor grado de resiliencia, la especie Mytilus edulis registra en Heshkaia cambios diacrónicos en el tamaño más pronunciados que los de Nacella sp. Aunque la información biométrica de estas últimas es muy parcial, posiblemente esto se deba a que los mejillones eran explotados más intensivamente, mientras que la baja proporción de lapas en los sitios indicaría que su recolección fue mínima e insuficiente como para afectar a las poblaciones locales de estos gasterópodos. Sin embargo, la longitud de valva de los mejillones presenta una tendencia de cambio muy irregular, con una disminución hacia los momentos medios de la secuencia, seguida por un incremento hacia los momentos más tardíos. Esta situación contrasta con un escenario típico de reducción diacrónica en el tamaño por sobreexplotación humana (Mannino y Thomas, 2002). El carácter discontinuo de las variaciones indicaría en cambio que a largo plazo el impacto antrópico no logró alterar la disponibilidad natural de los mejillones. En efecto, las elevadas tasas de desove y crecimiento de esta especie, así como el constante suministro de larvas desde aguas 


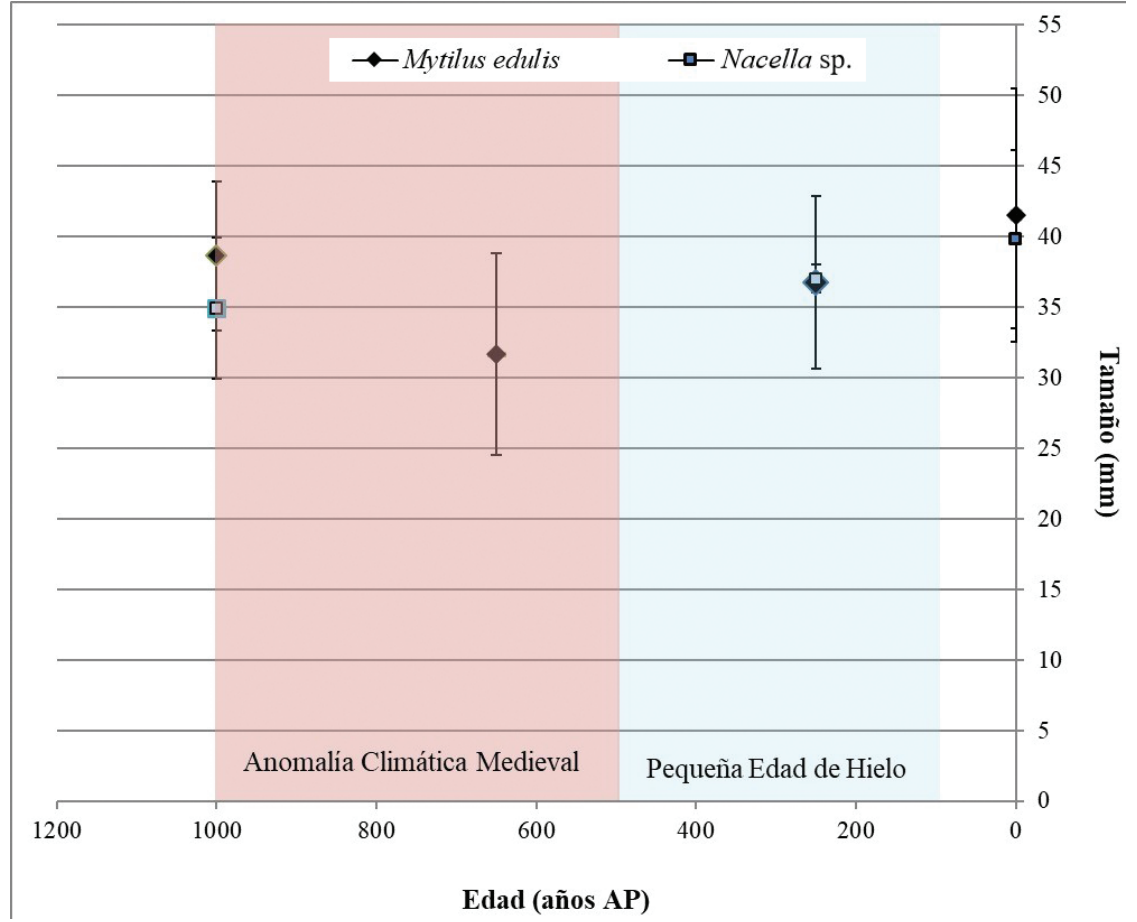

Figura 4. Tamaños medios de Mytilus edulis y Nacella sp. en diferentes momentos del Holoceno tardío.

más profundas y otros sectores de la costa habrían facilitado la rápida recuperación de sus poblaciones (Orquera y Piana, 1999).

Por otra parte, la amplia dispersión de medidas de longitud de los ejemplares Mytilus edulis en las muestras de todos los sitios es consistente con el correlato material de una estrategia recolectora del tipo stripping (Jones y Richman, 1995). Esto sugiere que durante el Holoceno tardío, los grupos locales practicaron la recolección indiferenciada de una amplia gama de tamaños disponibles en el ambiente intermareal. Dicha estrategia habría implicado, por definición, tasas de retorno globales inferiores a las que podrían obtenerse mediante el consumo exclusivo de los mejillones más grandes (Jones y Richman, 1995). Sumado a esto, los efectos negativos de la presión humana en el crecimiento de los moluscos habrían hecho que las recolecciones se efectuaran siempre bajo condiciones subóptimas de explotación, a partir de una oferta natural de mejillones que aún no presentaban un completo desarrollo y con un rendimiento calórico insuficiente.

\section{CONCLUSIONES}

En la localidad Heshkaia, la explotación de moluscos por los grupos cazadores-recolectores del
Holoceno tardío se centró en la recolección en racimos de bivalvos de bajo valor alimenticio pertenecientes a la especie Mytilus edulis. Su recolección se efectuó en un contexto de disponibilidad de ejemplares de tamaño subóptimo, producto de la fuerte presión recolectora ejercida en el ambiente intermareal. Estos resultados constatan una pauta local que coincide con los patrones previamente descriptos para el sitio Heshkaia 35 (Zangrando et al., 2017).

Por otra parte, cabe destacar algunas implicancias regionales de los resultados de este trabajo. En principio, la ausencia de un comportamiento optimizador de los retornos inmediatos de la recolección indica que el valor de los recursos malacológicos en la subsistencia de los cazadores-recolectores locales se definió por su abundancia natural y su accesibilidad, antes que por su valor nutricional. Esta observación refuerza la idea sostenida por Orquera y Piana (1999) con respecto al rol complementario de los moluscos en la economía de los grupos que habitaron las costas del canal Beagle durante la segunda mitad del Holoceno. El aporte calórico de estos invertebrados habría sido marcadamente inferior al de otros recursos de mayor porte disponibles en el espacio costero. No obstante, el bajo riesgo asociado a su explotación habría permitido la participación económica de niños y ancianos (Erlandson, 1988; Orquera y Piana, 2015, pp. 169-170).

En segundo lugar, las diferencias entre la composición malacológica de Heshkaia y la del canal Beagle central advierten sobre la existencia de algunas variantes locales en el aprovechamiento de la malacofauna de la región y en particular, de los taxones arqueológicamente menos representados, como Brachidontes sp. y gasterópodos. Este aspecto conduce a profundizar el conocimiento sobre los procesos de formación que explican la variabilidad espacial de los conjuntos arqueomalacológicos. 
Por último, si bien la rápida recuperación de las poblaciones locales de Mytilus edulis habría neutralizado los efectos del impacto antrópico, algunos aspectos conductuales de los cazadores-recolectores tardíos de Heshkaia también pudieron favorecer su continuidad biológica. La técnica de recolección en racimos (stripping) ha sido caracterizada como una estrategia conservativa que, al reducir la competencia intraespecífica, incrementa a largo plazo la productividad global de los bancos de mitílidos (Whitaker, 2008). Asimismo, las oscilaciones en el tamaño medio de los mejillones a lo largo del tiempo sugieren una pauta de ocupación del espacio de corto plazo que favorecería la recomposición de los bancos de mitílidos en los lapsos en los cuales no eran explotados. Este patrón de cambio oscilatorio ha sido detectado en las secuencias de ocupación de algunas localidades del canal Beagle central (Orquera y Piana, 2001) y sería consistente con un escenario regional de grupos cazadores-recolectores con alta movilidad a lo largo de las costas del canal Beagle (Orquera y Piana, 1999). En el futuro, esta información deberá corroborarse mediante líneas de estudio que aporten mayor precisión sobre las pautas temporales de explotación de moluscos, tales como los estudios de estacionalidad basados en el análisis de isótopos de oxígeno en los exoesqueletos de ejemplares arqueológicos de moluscos (Colonese et al., 2011).

\section{Agradecimientos}

A Francisco Zangrando, Augusto Tessone, Angélica M. Tivoli y Luis A. Orquera por la lectura crítica del manuscrito. A Germán Pinto Vargas por la ayuda brindada en el procesamiento de las muestras. A la Dra. Sandra Gordillo por su asesoramiento en la identificación de los restos de moluscos. A Martín Vázquez, María P. Martinoli y Ernesto Piana por sus valiosos aportes a este trabajo. A los evaluadores, cuyas sugerencias contribuyeron a fortalecer los argumentos del manuscrito. Este trabajo de investigación ha sido financiado por el proyecto PICT 2013- 1011 y PICT 2017-1230.

\section{REFERENCIAS CITADAS}

Alunni, D. V. y Zangrando, A. F. (2012). Primeros datos sobre el transporte, procesamiento y consumo de guanacos en la localidad arqueológica Heshkaia (sudeste de Tierra del Fuego, Argentina). Magallania, 40(1), 319-331.
Bettinger, R. L. y Malhi, R. (1997). Central place models of acorn and mussel processing. Journal of Archaeological Science, 24(10), 887-899.

Campbell, G. E. (2012). The archaeological potential of fragmentary remains of the Common Mussel, Mytilus edulis. (L.) [tesis de maestría inédita, University of Reading].

Codding, B. F., O'Connell, J. F. y Bird, D. W. (2014). Shellfishing and the colonization of Sahul: multivariate model evaluating the dynamic effects of prey utility, transport considerations and life-history on foraging patterns and midden composition. Journal of Island \& Coastal Archaeology, 9, 238-252.

Colonese, A. C., Camarós, E., Verdún Castelló, E., Giralt, S. y Rejas, M. (2011). Integrated archaeozoological research of shell middens: new insights into huntergatherer-fisher coastal exploitation in Tierra del Fuego. Journal of Island and Coastal Archaeology, 6, 235-254.

Erlandson, J. M. (1988). The role of shellfish in prehistoric economies: a protein perspective. American Antiquity, 53(1), 102-109.

Forcelli, D. O. (2000). Moluscos magallánicos. Guía de moluscos de Patagonia y Sur de Chile. Vázquez Manzini Editores.

Gordillo, S. (1995). Moluscos australes, una guía ilustrada. Bivalvos y caracoles de las costas del extremo sur de América. Zagier \& Urruty.

Gordillo, S., Brey, T., Beyer, K. y Lomovasky, B. J. (2015). Climatic and environmental changes during the middle to late Holocene in southern South America: a sclerochronological approach using the bivalve Retrotapes exalbidus (Dillwyn) from the Beagle Channel. Quaternary International Journal, 377, 83-90.

Hammond, H. (2015). Sitios Concheros en la Costa Norte de Santa Cruz: su estructura arqueológica y variabilidad espacial en cazadores recolectores patagónicos [tesis doctoral inédita, Universidad Nacional de La Plata].

Jones, T. L. y Richman, J. R. (1995). On mussels: Mytilus californianus as a prehistoric resource. North American Archaeologist, 16(1), 33-58.

Kidwell, S. M. (2002). Time-averaged molluscan death assemblages: Palimpsests of richness, snapshots of abundance. Geology, 30(9): 803-806.

Kidwell, S. M. (2013). Time-averaging and fidelity of modern death assemblages: Building a taphonomic foundation for conservation palaeobiology. Palaeontology, 56(3), 487-522. 
Langley, S., Guzman, L. y Ríos, C. (1980). Aspectos dinámicos de Mytilus chilensis (Hupé, 1840) en el estrecho de Magallanes. I. Distribución, densidad y disposición espacial en el intermareal. Anales del Instituto de la Patagonia, Serie Ciencias Naturales, 11, 319-332.

Lasta, M. L., Ciocco, N. F., Bremec, C. S. y Roux, A. M. (1998). Moluscos bivalvos y gasterópodos. En E. E. Boschi (Ed.), El Mar Argentino y sus recursos pesqueros, t. 2. Los moluscos de interés pesquero. Cultivos y estrategias reproductivas de bivalvos y equinoideos (pp. 115-142). Instituto Nacional de Investigación y Desarrollo Pesquero, Secretaría de Agricultura, Ganadería, Pesca y Alimentación.

Mannino, M. A., y Thomas, K. D. (2002). Depletion of a resource? The impact of prehistoric human foraging on intertidal mollusc communities and its significance for human settlement, mobility and dispersal. World Archaeology, 33(3), 452-474.

Martinoli, M. P. (2018). Modalidades de explotación, procesamiento y consumo de pinnípedos en la margen meridional de Tierra del Fuego [tesis doctoral inédita, Universidad de Buenos Aires].

Mason, R. D., Peterson, M. L. y Tiffany, J. A. (1998). Weighing vs. counting: measurement reliability and the California School of Midden Analysis. American Antiquity, 63(2), 303-324.

Menge, B. A., Chan, F. y Lubchenco, J. (2008). Response of a rocky intertidal ecosystem engineer and community dominant to climate change. Ecology Letters, 11(2), 151-162.

Obelic, B., Álvarez, A., Argullós, J. y Piana, E. L. (1998). Determination of water paleotemperature in Beagle Channel (Argentina) during the last $6000 \mathrm{yr}$ through stable isotope composition of Mytilus edulis shells. Quaternary of South America and Antartic Peninsula, 11, 47-71.

Oehrens Kissner, E. M. y Kroeck, M. A. (2005). Estimación de la talla de primera madurez sexual del mejillón, Mytilus edulis platensis (Mollusca: Bivalvia) en el Golfo San Matías (IBMP Serie Publicaciones IV), 21-44.

Ojeda, J., Rosenfeld, S., Marambio, J., Rozzi, R. y Mansilla, A. (2014). Patrones estacionales y espaciales de la diversidad de moluscos intermareales de bahía Róbalo, canal Beagle, Reserva de la Biosfera Cabo de Hornos, Chile. Revista de Biología Marina y Oceanografía, 49(3), 493-509.

Orquera, L. A. (1999). El consumo de moluscos por los canoeros del extremo sur. Relaciones de la Sociedad Argentina de Antropología, XXIV, 307-327.
Orquera, L. A. y Piana, E. L. (1999). Arqueología de la región del canal Beagle (Tierra del Fuego, República Argentina). Sociedad Argentina de Antropología.

Orquera, L. A. y Piana, E. L. (2000). Composición de conchales de la costa del canal Beagle (Tierra del Fuego, República Argentina)-primera parte. Relaciones de la Sociedad Argentina de Antropología, XXV, 249-274.

Orquera, L. A. y Piana, E. L. (2001). Composición de conchales de la costa del canal Beagle (Tierra del Fuego, República Argentina)-segunda parte. Relaciones de la Sociedad Argentina de Antropología, XXVI: 345-368.

Orquera, L. A. y Piana, E. L. (2015). La vida social y material de los Yámana. Ediciones Monte Olivia.

Pérez, S. A. (2018). Estrategias de explotación de moluscos durante el Holoceno tardío en la localidad de Heshkaia, Moat, sur de Tierra del Fuego [tesis de licenciatura inédita, Universidad de Buenos Aires].

Rosenfeld, S., Marambio, J. y Aldea, C. (2013). Comparación de ensambles de moluscos en dos sustratos intermareales de la cuenca central del Estrecho de Magallanes. Amici Moluscarum, 21(2), 7-18.

Stiner, M. C., Munro, N. D. y Surovell, T. A. (2000). The tortoise and the hare: small game uses, the broad spectrum revolution, and Paleolithic demography. Current Anthropology, 41(1), 39-73.

Verdún Castelló, E. (2014). Application of biometric analyses on shell middens of hunter-fisher-gatherer societies of Tierra del Fuego (Argentina). Archaeofauna, 23, 223-238.

Whitaker, A. R. (2008). Incipient aquaculture in prehistoric California?: long-term productivity and sustainability vs. immediate returns for the harvest of marine invertebrates. Journal of Archaeological Science, 35, 1114-1123.

Whitaker, A. R. y Byrd, B. F. (2014). Social circumscription, territoriality and the Late Holocene intensification of small-bodied shellfish along the California Coast. Journal of Island and Coastal Archaeology, 9(2), 150-168.

Zangrando, A. F., Tessone, A. y Vázquez, M. (2009). El uso de espacios marginales en el archipiélago fueguino: implicaciones de la evidencia arqueológica de Bahía Valentín. En M. Salemme, F. Santiago, M. Álvarez, E. Piana, M. Vázquez y E. Mansur (Eds.), Arqueología de la Patagonia. Una mirada desde el último confín (pp. 47-62). Utopías. 
La explotación de moluscos intermareales durante el Holoceno Tardío en la localidad de... Intersecciones en Antropología 21(1), enero-junio. 2020. ISSN-e 1850-373X

Zangrando, A. F., Alunni, D. V., Martinoli, M. P., Tivoli, A. M. y Piana, E. L. (2010). Arqueología de la región de Moat (Tierra del Fuego, Argentina): estudios preliminares en la localidad arqueológica Heshkaia. En J. R. Bárcena y H. Chiavazza (Eds.), Arqueología Argentina en el Bicentenario de la Revolución de Mayo, Actas XVII Congreso Nacional de Arqueología Argentina (pp. 2005-2010). Mendoza, Argentina: Facultad de Filosofía y Letras, Universidad Nacional de Cuyo; Instituto de Ciencias Humanas, Sociales y Ambientales, CONICET.
Zangrando, A. F., Borrazzo, K. B., Tivoli, A. M., Alunni, D. V. y Martinoli, M. P. (2013). El sitio Heshkaia 35: nuevos datos sobre la arqueología de Moat (Tierra del Fuego, Argentina). Revista del Museo de Antropología, 7(1), 11-24.

Zangrando, A. F., Pinto Vargas, G. y Tivoli, A. M. (2017). Decreased foraging return in shellfishing? Species composition and shell size of blue mussel (Mytilus edulis) from a Late Holocene site of the South Coast of Tierra del Fuego. Quaternary International, 427, 160-169. 
